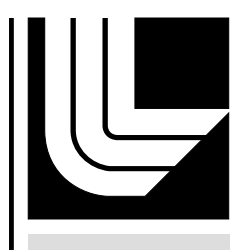

LAW RENCE LIVERMORE N A T IO N A L LABORATORY

\title{
UCRL-TR-227895
}

Looping through the Lamb Shift

A. U. Hazi

February 8, 2007 
This document was prepared as an account of work sponsored by an agency of the United States Government. Neither the United States Government nor the University of California nor any of their employees, makes any warranty, express or implied, or assumes any legal liability or responsibility for the accuracy, completeness, or usefulness of any information, apparatus, product, or process disclosed, or represents that its use would not infringe privately owned rights. Reference herein to any specific commercial product, process, or service by trade name, trademark, manufacturer, or otherwise, does not necessarily constitute or imply its endorsement, recommendation, or favoring by the United States Government or the University of California. The views and opinions of authors expressed herein do not necessarily state or reflect those of the United States Government or the University of California, and shall not be used for advertising or product endorsement purposes.

This work was performed under the auspices of the U.S. Department of Energy by University of California, Lawrence Livermore National Laboratory under Contract W-7405-Eng-48. 


\section{Looping through the Lamb Shift}

OMETIMES in science, a small measurement can have big ramifications. For a team of Livermore scientists, such was the case when they measured a small shift in the spectrum of extremely ionized atoms of uranium.

The measurement involves the Lamb shift, a subtle change in the energy of an electron orbiting an atom's nucleus. The precision of the Livermore result was 10 times greater than that of existing measurements, making it the best measurement to date of a complicated correction to the simplest quantum description of how atoms behave. The measurement introduces a new realm in the search for deviations between the theory of quantum electrodynamics (QED), which is an extension of quantum mechanics, and the real world. Such deviations, if discovered, would have far-reaching consequences, indicating that QED is not a fundamental theory of nature.

\section{A Test of QED}

The Lamb shift has nothing to do with sheep and everything to do with quantum theory, which deals with the world of the very small - atoms, electrons, neutrons, photons, and so on. According to quantum theory, energy is emitted and absorbed in tiny, discrete amounts, or quanta. When an electron changes from one energy level to another, it emits or absorbs a photon of light. This light can be observed through spectroscopy, and its frequency is directly proportional to the difference between the two energy levels.

Most of the Lamb shift can be explained by two basic QED phenomena: self-energy and polarized vacuum. The self-energy effect occurs when electrons subjected to electric and magnetic fields spontaneously and randomly emit photons and, within an incredibly short time span, reabsorb them. Through a technique called renormalization, QED theory provides a means to determine the energy of this electron self-interaction as a small, finite correction to the energy that binds the electron to the nucleus.

The polarized vacuum effect involves pairs of "virtual particles," one with a positive charge and one with a negative charge. Such pairs are created spontaneously from a vacuum in the presence of a positive or negative field and then are polarized, or aligned, by the field's charge. For example, say that a virtual electron-positron pair occurs in the presence of a positively charged ion. The virtual electrons, drawn to the positive charge,

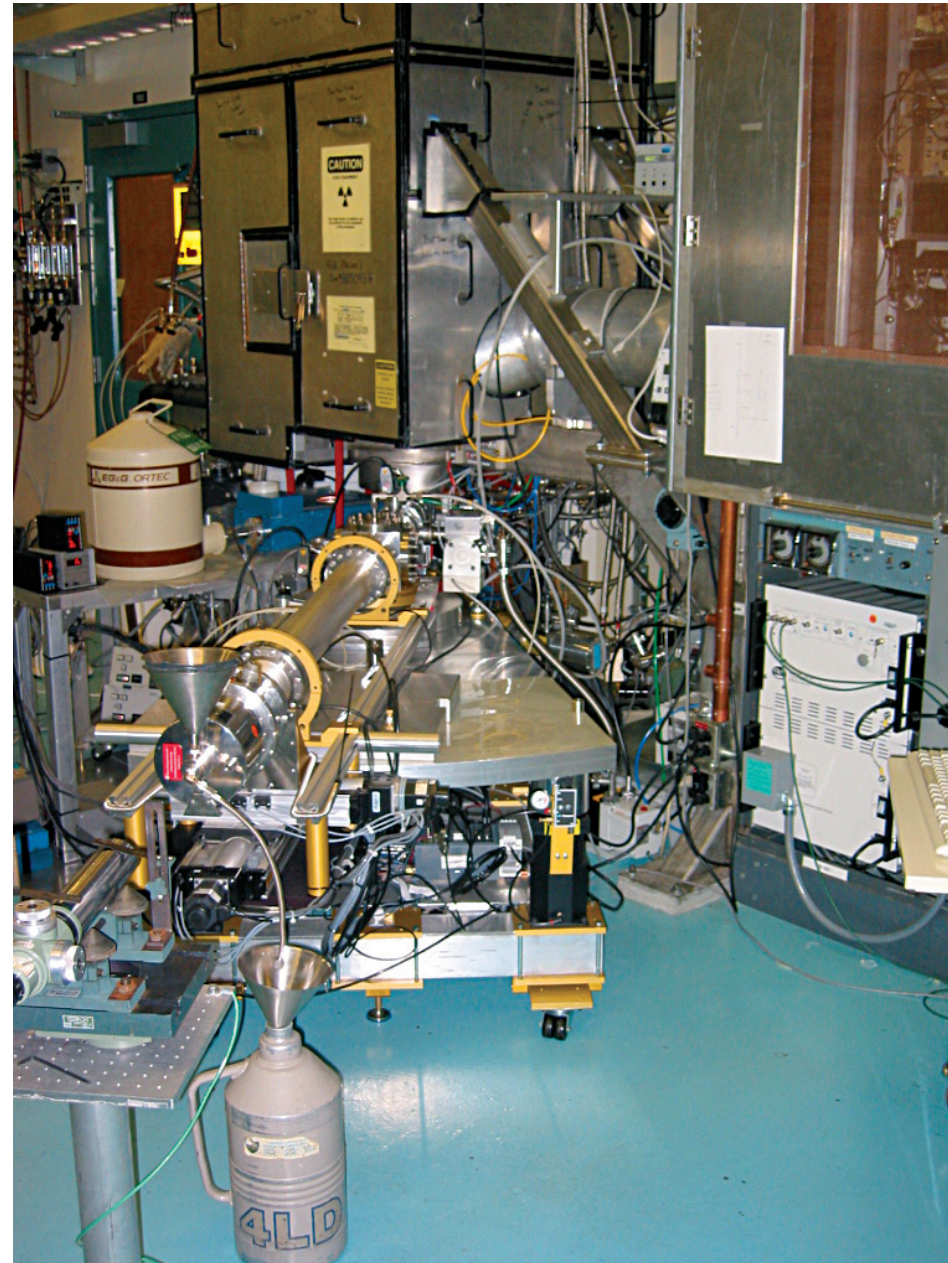

SuperEBIT, Livermore's electron-beam ion trap, can create and trap highly charged ions.

tend to align closer to the ion's nucleus than virtual positrons, which are repelled by the positive charge. Thus, an electron that orbits an ion travels with fickle companions - swarms of virtual photons and electron-positron pairs - randomly winking in and out of existence.

The Lamb shift is a tiny difference in the energy of an ion's electron between two quantum states that are otherwise identical except for the shape of the electron's orbit around the nucleus. Because of this dissimilarity in the electron's orbit in the two states, the electron interacts slightly differently with virtual particles - photons, positrons, and electrons - that appear and disappear in quantum fluctuations. The resulting energy difference, called the Lamb shift, can be measured when the electron jumps from one energy level to another.

Theory also predicts that even smaller, higher-order contributions to the Lamb shift exist. For the second-order Lamb shift, virtual 
photons and electron-positron pairs interact with themselves and each other to generate two loops. (See the figure below.)

"For instance," says Livermore physicist Peter Beiersdorfer, "a photon could be emitted, and, before it is reabsorbed, another photon could be emitted or an electron-positron pair created. In a another instance, an electron-positron pair could be created, and the virtual electron from that pair could emit a photon, which is quickly absorbed." In all, 10 second-order interactions are possible. (See the background image on p. 3.) Measuring the higher-order correction to the spectrum with sufficient precision would validate the model.

\section{Uranium Ion Takes the Field}

Previous experiments measured QED effects on hydrogen, which has a single electron but a very weak field. However, Beiersdorfer's team, which was funded by the Laboratory Directed Research and Development Program, decided to study the uranium atom, which has 92 electrons. "The stronger the electric and magnetic fields experienced by the electron, the stronger or more visible the interactions should be," says Beiersdorfer. "The electric field of a uranium ion is a million times stronger than that of hydrogen, which makes QED effects that much more pronounced."

Another advantage is that scientists know more about the size of the uranium atom's nucleus than they do about that of the hydrogen atom. Knowing the size of the nucleus is important in making a precise measurement, particularly when it is extended to many decimal places. A problem with hydrogen is the high degree of uncertainty in the size of its nucleus, the proton. Three measurements for the proton's radius are often quoted, but the error bars of the three do not overlap, thus forming a comparatively large band of uncertainty. "The precision in QED measurements of hydrogen has hit a wall," says Beiersdorfer, "because the measurements now outstrip the precision with which the hydrogen nucleus is known."

To measure highly charged uranium, the Livermore team used SuperEBIT, an electron-beam ion trap developed by Laboratory scientists. (See $S \& T R$, November 2000, pp. 19-21; June 2002, pp. 20-24.) SuperEBIT uses a tightly focused and energy-tunable electron beam to create and trap highly charged ions. This beam, which has a current density of up to 5,000 amperes per square centimeter, passes through a series of three drift tubes to create ions of the element being studied. These positively charged ions are confined radially because they are attracted to the center of the electron beam. Voltages applied at each end of the drift-tube series trap the ions axially. When the beam's electrons collide with an ion, they strip electrons off the ion until the energy required to remove the next electron is higher than the beam's energy. Once created, the highly charged ions are held while the scientists record the spectra.

SuperEBIT is the only ion source that can create such highly charged ions at rest. Other sources produce highly charged ions; however, because they use accelerators, these sources increase an ion's velocity to extremely high energies.

For the Lamb shift experiments, the team stripped off 89 of the 92 electrons in each uranium atom, leaving uranium ions with only three electrons bound to each nucleus. High-resolution spectrometers developed at Livermore then recorded the light emitted by the electrons of these lithiumlike uranium atoms. Spectra were recorded for about two months to collect enough data for analysis. "The count rate for this event is quite low, something like four to five counts per hour," says Beiersdorfer, "and the signal is very faint." (a)

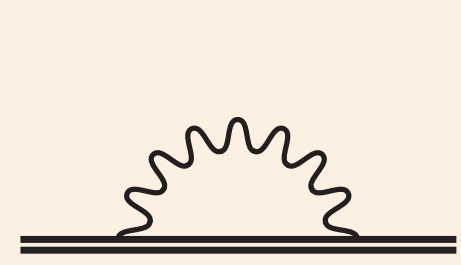

(b)

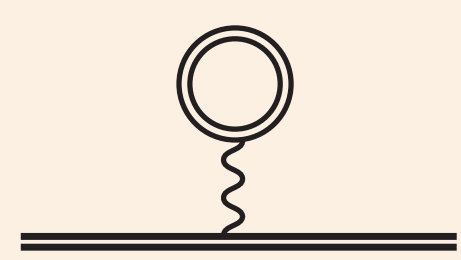

(c)

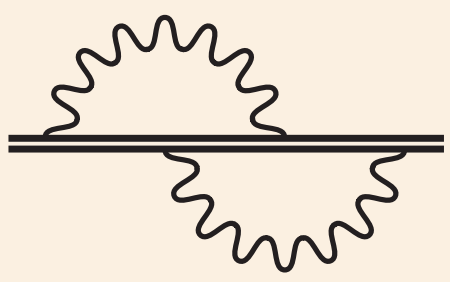

(d)

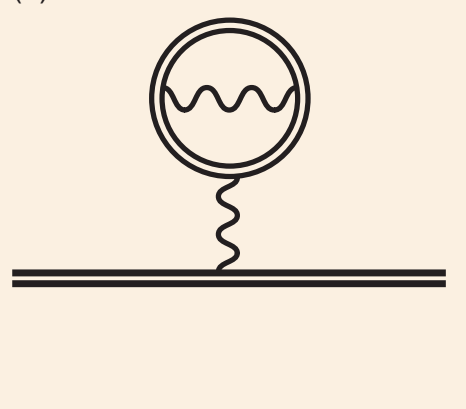

Feynman diagrams, which are used to illustrate quantum theory, show contributions to the Lamb shift. Double lines represent bound electrons, wavy lines represent virtual photons, and double circles represent virtual electron-positron pairs. When an electron is subjected to electric and magnetic fields, it can (a) emit and reabsorb a virtual photon or (b) emit and reabsorb a virtual electron-positron pair. These interactions involve a single loop of virtual particles and are the first-order contribution to the Lamb shift. More complex interactions are possible, in which two or more loops of virtual particles are generated. Examples of a two-loop contribution to the Lamb shift are shown in (c) and (d). In (c), an electron emits one photon and then, soon after, another, so that the two overlap in time. In (d), when a virtual electron-positron pair is emitted, the virtual electron emits a photon that is reabsorbed. 


\section{Shedding Light on QED}

The team measured the contribution of both the one- and two-loop Lamb shifts to the observed spectrum and improved the existing experimental precision by nearly an order of magnitude. In doing so, the team surpassed the precision achieved by researchers at the Heavy Ion Research Institute in Germany.

"These were the first measurements of the two-loop Lamb shift in a strongly bound system," says Beiersdorfer. The results, which were chosen as a 2005 highlight by the American Physical Society's News Bulletin, are stimulating new calculations in QED theory. Laboratory physicists K. T. Cheng and Mau Chen, in collaboration with Jonathan Sapirstein from Notre Dame University, are making calculations, which they will compare to the experimental results. Livermore physicist Elmar Träbert, together with Beiersdorfer, is leading a study to measure highly stripped bismuth. Bismuth's electric field is weaker than uranium's, but its nuclear magnetic field is very strong. Träbert's study, which includes Livermore physicists Hui Chen, Greg
Brown, and Daniel Thorn, will examine the processes that create such strong magnetic fields inside the nucleus.

"In QED experiments such as these, we are looking for one of two outcomes," says Beiersdorfer. "If the measurements support theoretical calculations, they reaffirm that the theory fits reality. If the measurements and calculations do not agree, scientists are faced with a mystery to solve, because the discrepancies show us that we have more to learn."

-Ann Parker

Key Words: electron-beam ion trap, ions, polarized vacuum, quantum electrodynamics (QED), quantum fluctuations, quantum mechanics, selfenergy, spectroscopy, SuperEBIT, two-loop Lamb shift, uranium, virtual particles, virtual photons.

For further information contact Peter Beiersdorfer (925) 423-3985 (beiersdorfer1@IInl.gov).
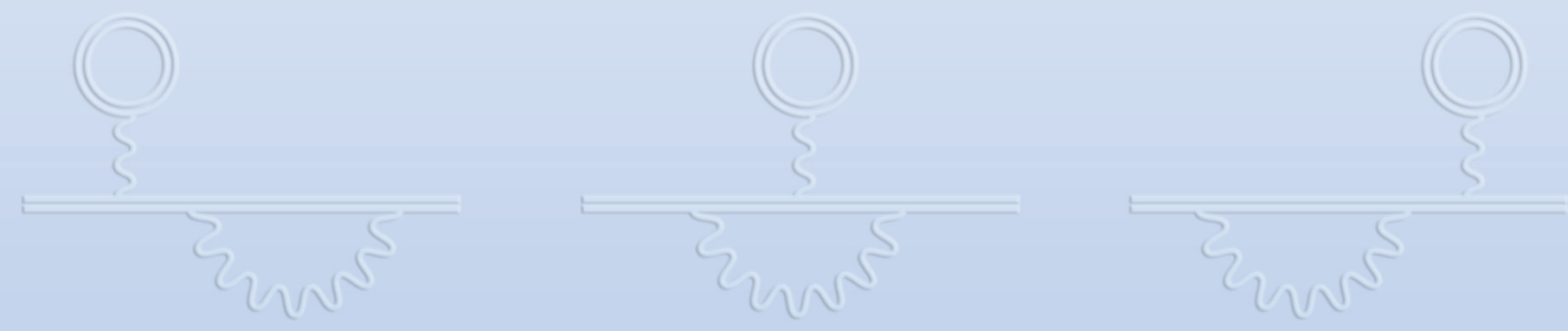